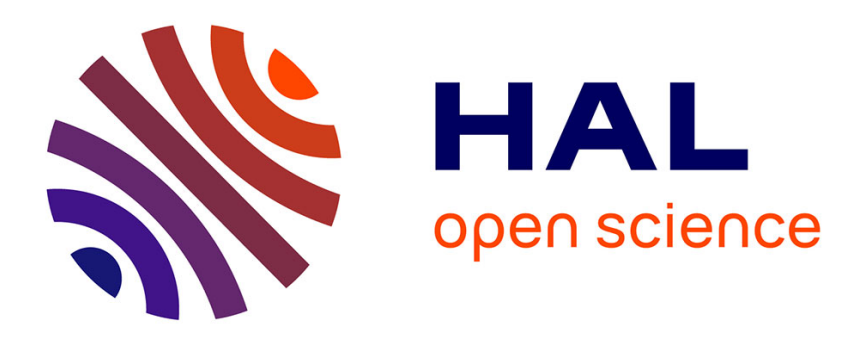

\title{
Permeability of Self-Affine Aperture Fields
}

Laurent Talon, Harold Auradou, Alex Hansen

\section{To cite this version:}

Laurent Talon, Harold Auradou, Alex Hansen. Permeability of Self-Affine Aperture Fields. Physical Review E: Statistical, Nonlinear, and Soft Matter Physics, 2010, 82 (4), pp.046108. 10.1103/PhysRevE.82.046108 . hal-00472230

\section{HAL Id: hal-00472230 \\ https://hal.science/hal-00472230}

Submitted on 18 Apr 2010

HAL is a multi-disciplinary open access archive for the deposit and dissemination of scientific research documents, whether they are published or not. The documents may come from teaching and research institutions in France or abroad, or from public or private research centers.
L'archive ouverte pluridisciplinaire $\mathbf{H A L}$, est destinée au dépôt et à la diffusion de documents scientifiques de niveau recherche, publiés ou non, émanant des établissements d'enseignement et de recherche français ou étrangers, des laboratoires publics ou privés. 


\title{
Permeability of Self-Affine Aperture Fields
}

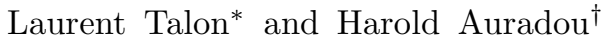 \\ Univ. Pierre et Marie Curie-Paris6, Univ. Paris-Sud, CNRS, \\ Lab. FAST, Bât. 502, Campus Univ., Orsay, F-91405, France.
}

\author{
Alex Hansen \\ Department of Physics, Norwegian University of Science and Technology, N-7491 Trondheim, Norway
}

(Dated: April 18, 2010)

\begin{abstract}
We introduce a model that allows for the prediction of the permeability of self-affine rough channels (one-dimensional fracture) and two-dimensional fractures over a wide range of apertures. In the lubrication approximation, the permeability shows three different scaling regimes. For fractures with a large mean aperture or an aperture small enough to the permeability being close to disappearing, the permeability scales as the cube of the aperture when the zero level of the aperture is set to coincide with the disappearance of the permeability. Between these two regimes, there is a third regime where the scaling is due to the self-affine roughness. For rough channels, the exponent is found to be $3-1 / H$ where $H$ is the Hurst exponent. For two-dimensional fractures, it is necessary to introduce a new equivalent aperture $b_{c}$ to make the scaling regime apparent. $b_{c}$ is defined as the hydraulic aperture of the most restrective barrier crossing the fracture normal to the flow direction. This regime is characterized by an exponent higher than for the one-dimensional case: it is 2.25 for $H=0.8$ and 2.16 for $H=0.3$.
\end{abstract}

\section{INTRODUCTION}

In the last decades important research efforts from different communities have been devoted to upscaling the permeability of fractures. One of the practical issues, for instance, for long term sequestration or for geotechnical purposes is to predict the behavior of the permeability under changing mechanical conditions [1]. To uncover fundamental physical properties of transport phenomena in fractures, laboratory tests on rock samples [2, 3] or on modelled fractures [- 4 as well as numerical modelling [5] have been carried out. These studies have reported non-trivial relations between fracture aperture and the measured permeability. For large mean distance between the halves, the permeability is find to scale with the cube of this distance. In this limit, the fracture can be viewed as consisting of two parallel flat walls [3]. But, as soon as the halves are brought closer together, deviations from this cubic law due to the surface roughness are seen [6, 7. In the recent years, various theoretical models based on statistical averages, weak disorder perturbation expansions or mean field approximations have been tested to evaluate these deviations 8 16]. In spite of much invested work, most of the foregoing developments break down if contact zones exist in the fracture. When the fracture halves are brought even closer, all the fluid is finally forced to pass a single strait — or bottle neck connecting the inlet and the outlet. Following the work of Ambegoakar et al ("AHL") [17], the permeability of the entire fracture is then controlled by the permeability

\footnotetext{
*talon@fast.u-psud.fr

†auradou@fast.u-psud.fr

¥Alex.Hansen@ntnu.no
}

of the bottle neck [18, 19]. When the fracture is further opened percolating channels arise. The permeability is not controlled anymore by the bottle neck since the flow may bypass this region. In a previous study, we have extended the bottle neck effect by introducing the concept of critical barrier 20. In the present paper, we improve the method by taking into account secondary bottle necks. Our approach allows us to identify three regimes: the "AHL" regime (close to percolation), the cubic law regime for large mean aperture and an intermediate non-linear regime where the permeability is controlled by the successive critical constrictions. In the first section, Sec. II, we derive an extension of the bottle neck concept for flow in one-dimensional (1D) rough channels. In section III, we extend the critical path analysis to twodimensional (2D) fractures. We assume an aperture field which is the free space between a flat and a rough surface of height $h(x, y)$. Hence, the aperture is defined as $h(x, y)+a$ where $a$ is the aperture measured from the percolation point, i.e., for $a \leq 0$ there is no conducting channels and the permeability is zero. As soon as $a>0$, there is permeability. Possible contacts between the surfaces is also considered and flow is assumed to take place only in open voids of the fracture where $a(x, y)>0$. Places where the aperture is negative are considered in contact and their corresponding aperture $h(x, y)+a$ is set to 0 . The fracture aperture is change by moving apart the mean planes of the two surfaces and its permeability is then analytically (when the field is $1 \mathrm{D}$ ) or numerically computed (for 2D fields) by assuming that the Reynolds equation holds locally [21].

In the present work, we consider aperture fields with self-affine correlations, which are known to characterize natural fractures 222 24]. Such aperture fields have a two-point function $p_{2}(\Delta h, \Delta \vec{r})$, giving the probability density to find a height difference $\Delta h$ over a distance $\Delta \vec{r}$, 
that show the invariance

$$
\lambda^{H} p_{2}\left(\lambda^{H} \Delta h, \lambda \Delta \vec{r}\right)=p_{2}(\Delta h, \Delta \vec{r}),
$$

where $H$ is the Hurst or roughness exponent.

\section{ONE-DIMENSIONAL SYSTEMS}

We start by considering one-dimensional flow. In this case, the fracture field is assumed invariant in the $y$ direction and the flow occurs is a rough channel with local aperture $h(x)+a$. Flow is totally stopped as soon as the two surfaces come into contact, leading here to $\min _{x} h(x)=0$. The permeability $K$ of this onedimensional aperture field is, in the lubrication, limit given by the integral

$$
\frac{L}{K}=\int_{x_{0}}^{x_{L}} \frac{d x}{k(h(x)+a)^{3}},
$$

where $k$ is a constant and $x_{L}-x_{0}=L$, the length of the system. When the rough profile is discretized over a length $\Delta$, so that $h(x) \rightarrow h_{k}$, Eq.(2) becomes:

$$
\frac{L}{K}=\sum_{0}^{L / \Delta} \frac{\Delta}{k\left(h_{k}+a\right)^{3}} .
$$

Before considering self-affine correlations in $h(x)$, we investigate the simpler case when there are no spatial correlations. This is done in order to introduce the concepts that will be central in the following.

\section{A. Uncorrelated Aperture Fields}

When the aperture field has no spatial correlations, we use order statistics combined with Eq. (2) to determine the scaling properties of the permeability. The aperture field is fully characterized by the probability density $p(h)$ and its cumulative probability is $P(h)=\int_{0}^{h} d h^{\prime} p\left(h^{\prime}\right)$. By taking the advantage that in Eq.(2) the occurrence order of the apertures do not matter, we may therefore order the $h(x)$ distribution in ascending order. The ordering transformation is $h(x) \rightarrow h[\xi]=h(x[\xi])$, where $h\left[\xi_{1}\right] \leq$ $h\left[\xi_{2}\right]$ if $\xi_{1} \leq \xi_{2}$. We define $\bar{h}[\xi]$ as the average of $h[\xi]$ over an ensemble of realizations. From order statistics [25], we then have that

$$
P(\bar{h}[\xi])=\frac{\xi}{L}
$$

Note that we have - by definition $-\bar{h}[0]=0$ and $\left\langle\max _{x \in[0, L]} h(x)\right\rangle=\bar{h}[L]$, where $\langle\cdots\rangle$ refers to an ensemble average.

The average inverse permeability is then given by the expression

$$
\frac{L}{K}=\int_{0}^{L} \frac{d \xi}{k(\bar{h}[\xi]+a)^{3}}=\int_{0}^{L} \frac{d \xi}{k\left(P^{-1}(\xi / L)+a\right)^{3}},
$$

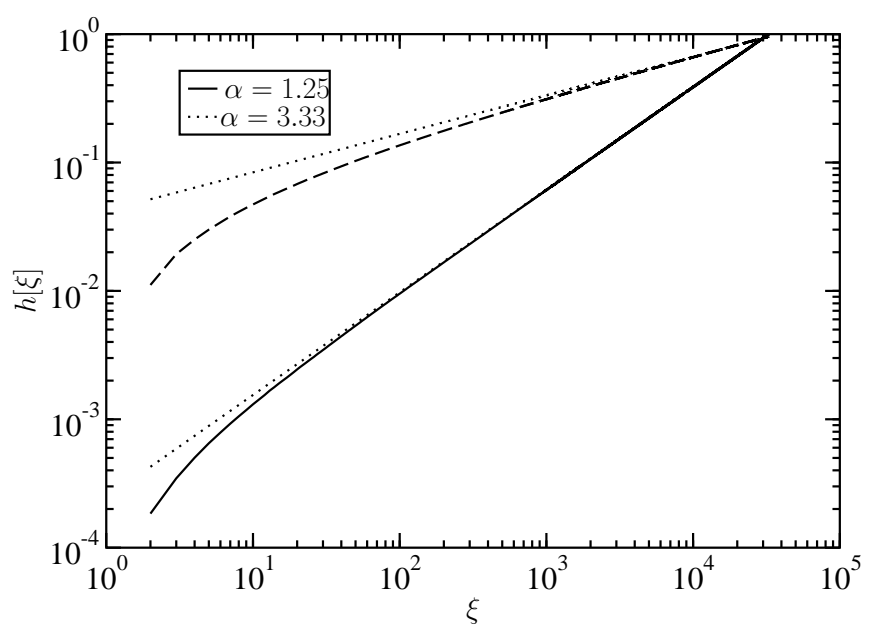

Figure 1: Ordered sequences $h[\xi]$ from an uncorrelated noise distributed on the interval $[0,1]$ according to the power law $p(h) \sim h^{\alpha-1}$ with exponent exponent $\alpha=1.25$ (solid line) and 3.33 (dashed line). The data have been averaged over 1000 samples and each sample has length $2^{15}$. The dotted lines have slopes $0.8=1 / 1.25$ and $0.3=1 / 3.33$ respectively.

In the situation where the aperture field is distributed according to a power law, $p(h) \propto h^{\alpha-1}$, where $\alpha>0$, bounded above by $\delta$, the cumulative probability is given by $P(h)=(h / \delta)^{\alpha}$. This leads to :

$$
\bar{h}[\xi]=P^{-1}(\xi / L)=\frac{\delta}{L^{1 / \alpha}} \xi^{1 / \alpha} .
$$

This result is illustrated in Fig. 1 where the ordered sequence $\bar{h}[\xi]$ is shown as a function of $\xi$ for $\alpha=1.25$ and 3.33 respectively.

The permeability of a power-law distributed aperture field is then given by the integral

$$
\frac{L}{K}=\int_{0}^{L} \frac{d \xi}{k\left(\left(\delta / L^{1 / \alpha}\right) \xi^{1 / \alpha}+a\right)^{3}} .
$$

We show in Fig. 2, the permeability $K$ as a function of the opening $a$ for $\alpha=1.25$ based on lubrication limit expression Eq. (3) together with the solution of Eq. (7).

As is apparent in Fig. 2, there are three power law regimes. We identify them in the following. By introducing the notation

$$
I(y)=\frac{1}{y^{\alpha}} \int_{0}^{y^{\alpha}} \frac{d \xi}{\left(\xi^{1 / \alpha}+1\right)^{3}},
$$

Eq. (7) may be written

$$
\frac{1}{K}=\frac{I((\delta / a))}{k a^{3}}
$$

Depending on the value of the ratio $a / \delta, I$ displays two scaling regimes. In the first limit, when $a / \delta \rightarrow \infty$, corresponding for instance to a widely open fracture or to 


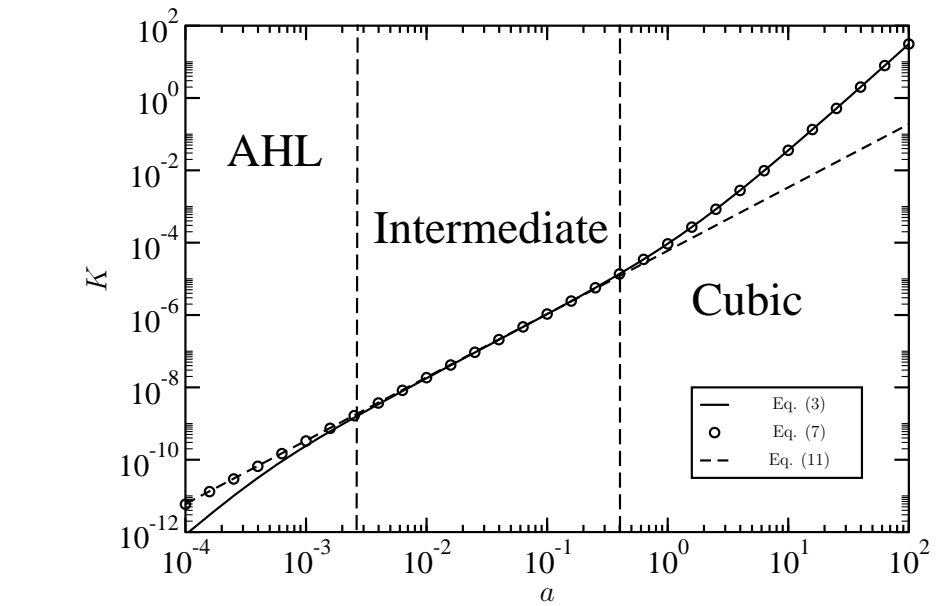

Figure 2: Permeability $K$ averaged over 1000 fields of length $2^{15}$ with $h$ distributed according to a power law $p(h) \sim h^{\alpha-1}$ on the unit interval, where $\alpha=1.25$. Solid line and open circles show respectively the permeability $K$ calculated from Eqs. (3) and (7) respectively as a function of minimum opening $a$. The three scaling regimes are delimited by vertical lines. The dashed line represent $a^{3-\alpha}=a^{1.75}$.

a fracture with small wall roughness, $I(\delta / a)$ tends to 1 and Eq.(9) becomes

$$
K=k a^{3}
$$

In this regime, the permeability follows the classical cubic law. In the other limit, when $a / \delta \rightarrow 0$, i.e., when the fracture is closed we deduce from Eq. (7) that the inverse permeability behaves as

$$
\frac{1}{K}=\frac{I_{\infty}}{k a^{3}}\left(\frac{a}{\delta}\right)^{\alpha} \sim \frac{1}{a^{3-\alpha}},
$$

where

$$
I_{\infty}=\int_{0}^{\infty} \frac{d \xi}{\left(\xi^{1 / \alpha}+1\right)^{3}} .
$$

When $\alpha=1.25, I_{\infty} \approx 0.52065$. Hence, in this regime, the permeability shows a non-linear variation with the fracture aperture with an exponent $3-\alpha$.

The relation given by Eq.(11) breaks down for small enough $a$ when the system is discretized, $h(x) \rightarrow h_{k}$. When discretized, the Eq. (7) reads

$$
\frac{L}{K}=\sum_{0}^{L / \Delta} \frac{\Delta}{k\left(W /(L / \Delta)^{1 / \alpha} k^{1 / \alpha}+a\right)^{3}} .
$$

For small enough $a$, the first term in the sum will dominate and the permeability is then given by

$$
K=\frac{L}{\Delta} k a^{3}
$$

This third scaling regime is visible in Fig. 2 for the permeability calculated from discretized fields $h_{k}$, where

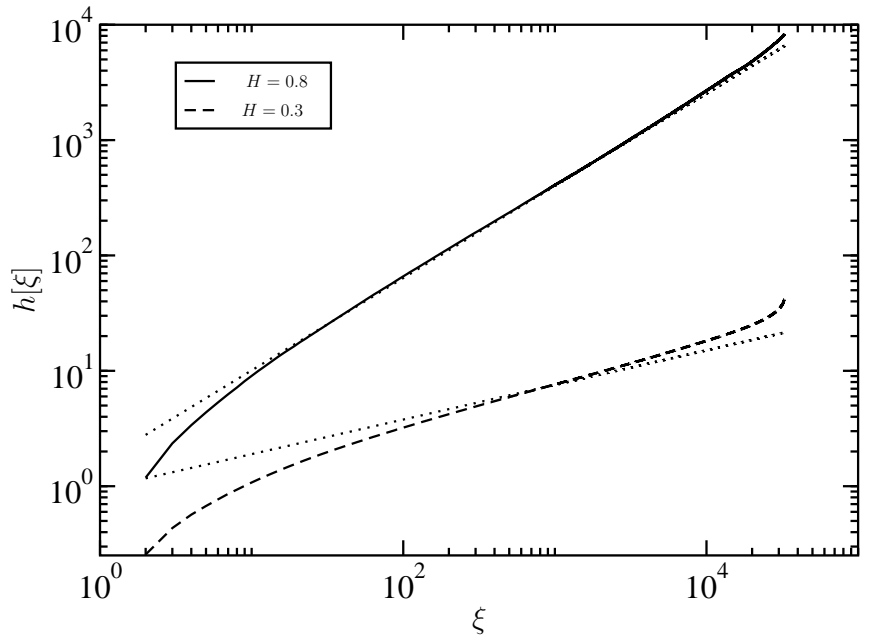

Figure 3: Ordered and averaged sequence $\bar{h}[\xi]$ based on 1000 self-affine $h(x)$ with Hurst exponents equal to 0.8 and 0.3 respectively. The data consists of 1000 samples of length $2^{15}$. The straight lines are $\xi^{0.8}$ and $\xi^{0.3}$ respectively.

$1 \leq k \leq 2^{15}$. The analytical calculation based on Eq. (7) (dashed line in Fig. 2) does not exhibit such regime.

In this paragraph, we have demonstrated that for uncorrelated powerlaw distributed aperture field three scaling regimes exists: For small $a / \delta, K \sim a^{3}$ for intermediate $a / \delta, K \sim a^{3-\alpha}$, and for large $a / \delta, K \sim a^{3}$ again. The next paragraph extends this feature to self-affine correlated fields.

\section{B. Self-Affine Correlations in the Aperture Field}

As the previous case, the zero level of the self affine field $h(x)$ is adjusted so that $\min _{x} h(x)=0$. Because of correlations, the eq. (化) cannot be straightforwardly used to compute the permeability. Hence, we conjecture that the averaged ordered sequence of $h$ is given by

$$
\bar{h}[\xi]=c \xi^{H},
$$

where $c$ is a prefactor. We test this conjecture in Fig. 3. For $H$ larger than 0.5 , the scaling predicted by (15) falls onto the numerical observation, for $\xi$ larger than 50 (similarly with the powerlaw distribution, see Fig. 1). However, for smaller values of $H$, the deviation becomes larger, see Fig. 3. For the 1D situation, we shall only consider a Hurst exponent $H>0.5$.

We now combine Eq. (15) with Eq. (5) to calculate the permeability,

$$
\frac{L}{K}=\int_{0}^{L} \frac{d \xi}{k\left(c \xi^{H}+a\right)^{3}} .
$$

We show in Fig. 4 the permeability gotten from calculation of Eq. (3) and from Eq. (16).

Since Eqs. (16) and (7) are formally identical, the scaling analysis presented in Eqs. (8) to (10) is the same for 


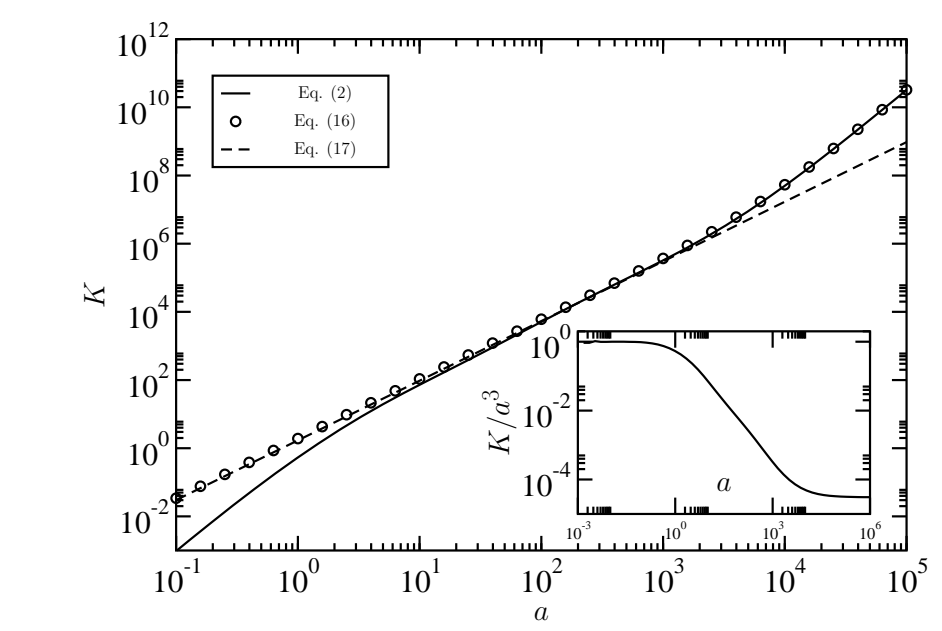

Figure 4: Permeability $K$, defined in Eq. (3) as a function of minimum opening $a$ for 1000 self-affine fields $h(x)$ of length $2^{15}$ with $H=0.8$. We also show the integral Eq. (16) as a function of $a$. The straight dotted line is the power law $a^{3-1 / H}=a^{1.75}$, Eq. (17). The inset shows the normalized permeability $K / a^{3}$.

the self-affine case as for the power-law distributed and uncorrelated functions when the exponent $\alpha$ is substituted for $1 / H$. Hence, for intermediate minimum apertures, $a$, we find the scaling

$$
K=\frac{k}{I_{\infty}} L a^{3-1 / H}
$$

We note that the permeability is in this intermediate regime proportional to the length $L$. This is different from the prediction of Roux et al. [26] giving $K \sim L^{3 H}$ in the same regime. This calculation was based on the assumption that $K \sim W^{3}$ where $W$ is the average aperture when the fracture surfaces are close to contact. The self affinity then gives $W \sim L^{H}$ and $K \sim L^{3 H}$ follows. However, Eq. (17) shows that even though the fracture opening $a$ is large enough so that the $\min _{x} h(x)$ region no longer dominates, $W$ does not enter the expression and the permeability is proportional to $L$ rather than $L^{3 H}$.

For small enough $a$, the region around the minimum aperture dominates and the permeability is given by Eq. (14). This was noted by noted by Gutfraind and Hansen 27] in their numerical study based on lattice gas automata.

\section{TWO DIMENSIONAL APERTURE FIELDS}

In going from one to two dimensions, i.e., when the aperture field is a function of points in a plane $(x, y)$ rather than only $x$, the concept of the narrowest constriction needs to be redefined. In one dimension, the narrowest constriction is the point along the fracture where there is first contact between the two halves. This definition does not work in two dimensions. The point at

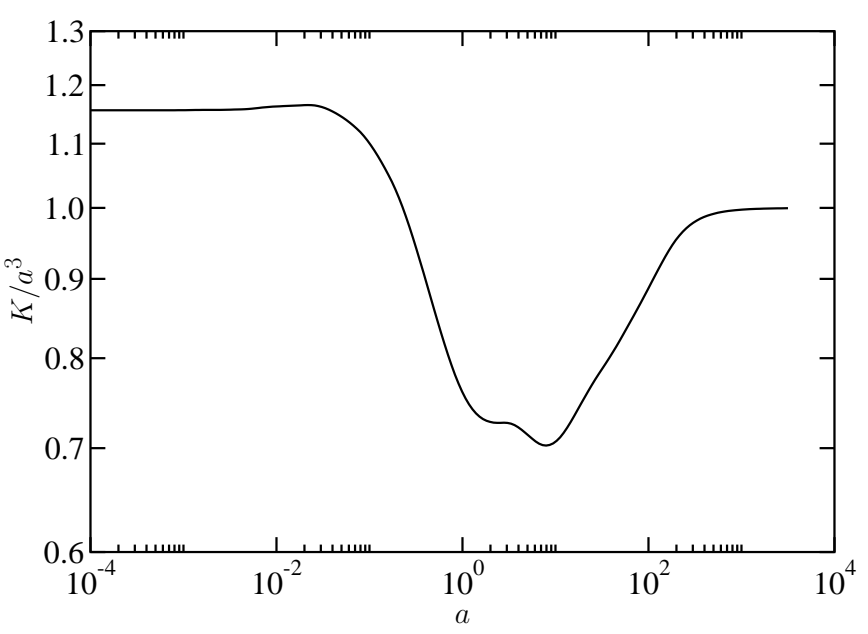

Figure 5: Permeability $K / a^{3}$ as a function of opening $a$ for 100 self-affine samples of size 1024 . For small and large $a$, $K \sim a^{3}$, but with different prefactors.

which there is first contact will have little influence in this case, as the flow simply goes around it. And, if the two halves are brought further into contact, deformation occurs. In this work, deformations are modelled as follow: First, the top surface $h(x, y)$ is adjusted such that $\min _{(x, y)} h(x, y)=0$. The aperture then define, after a vertical shift $u$, by $u(x, y)=u+h(x, y)$. There are contacts where $u(x, y)<0$. This is taken into account by defining

$$
n(x, y)=\max _{(x, y)}(u(x, y), 0) .
$$

Hence, by replacing $h(x, y)+u$ by $n(x, y)$ we model in a simple way the possible overlaps of the walls.

In one dimension, the aperture of the most narrow constriction is $a$. When $a$ approaches zero, the permeability decreases and reaches zero for $a=0$. We wish to define the aperture in the same way in two dimensions, namely $a$ is the aperture for which the permeability reaches zero. When the aperture is close to this value, the permeability is controlled by a single strait as argued in a different context by Ambegaokar et al. [17. The min-max algorithm of Hansen and Roux [28, 29] is used to identify the position of this particular point. First, we identify the minimum height along each pathes connecting the inlet to the outlet. The highest of the minimum height gives then the minimal vertical shift for which flow occurs. We will note $a$ this height. Note, using this method, one can also localize the percolation point that according to Ambegaokar et al. [17] should control the permeability.

Figure 5 shows the permeability, computed by solving the Kirchhoff equations, as function of the aperture $a$. The normalization of the permeability by $a^{3}$ highlights two plateaus for respectively small and large $a$. They correspond to the two cubic regimes already observed in the one-dimensional case.

In the intermediate regime, however, the normalized permeability does not show a power law in contrast to 


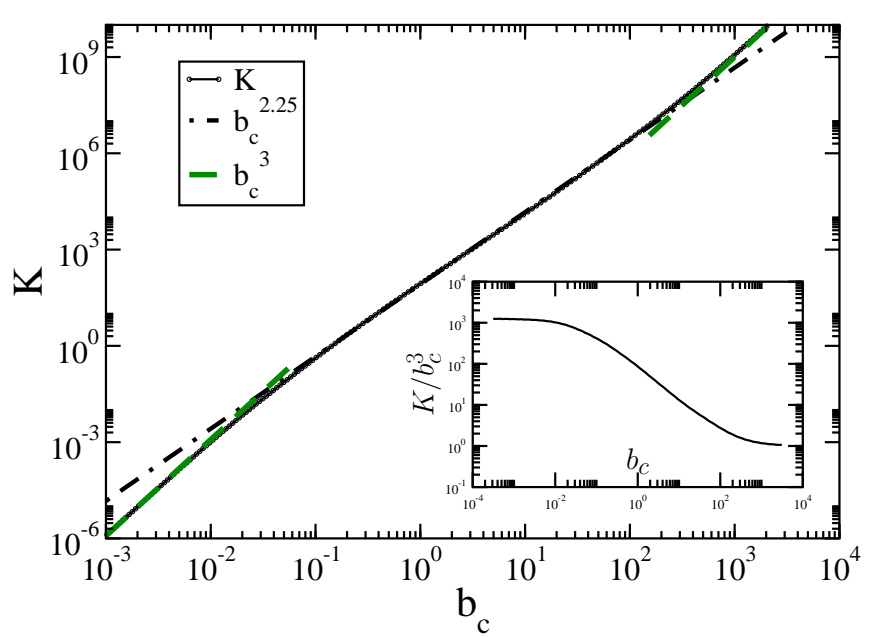

Figure 6: Permeability $K$ as a function of $b_{c}$ for 100 self-affine samples with $H=0.8$ of size $1024 \times 1024$. The straight lines are $b_{c}^{2.25}$ and $b_{c}^{3}$ respectively.

the one-dimensional case, see inset of Fig. A. This hints at the aperture $a$ does not correspond to the aperture defined in the one-dimensional case. We now identify the proper variable for this. In a recent paper, Talon et al. 20] replaced the one-dimensional notion of the "most narrow constriction" by the "most restrictive path" in two dimensions. If $\mathcal{C}$ is one out of all possible paths that cuts across the sample between the two edges parallel to the average flow direction, we may assign an "effective permeability" to it as the integral of $n^{3}(x, y)$ along $\mathcal{C}$. We then identify the path with the smallest effective permeability.

$$
b_{c}=\frac{1}{L}\left[\min _{\mathcal{C}} \int_{\mathcal{C}} d \vec{\ell} \cdot \vec{e}_{\perp} n^{3}(\vec{\ell})\right]^{1 / 3},
$$

where $\vec{e}_{\perp}$ is a unit vector pointing in the direction orthogonal to the average flow direction. When $a<<1, b_{c}$ will essentially be equal to $a$ as the only opening along the most restrictive path will be the "AHL" strait. However, for larger values of $a$, they will no longer coincide. As $a$ is increased even further, they again approach each other.

We show in Fig. 6 permeability $K$ as a function of $b_{c}$ as defined in Eq. (19). There are the small and large $b_{c}$ regimes where $K \sim b_{c}^{3}$. However, now there is also an intermediate regime where there is power law behavior,

$$
K \sim b_{c}^{2.25 \pm 0.02}
$$

for $H=0.8$. For surfaces with $H=0.3$, we find an exponent $2.16 \pm 0.02$.

In order to understand where this intermediate power law regime comes from, we generalize the concept of $b_{c}$. Assume now that we are no longer looking for the most restrictive path for the entire sample, but also for other

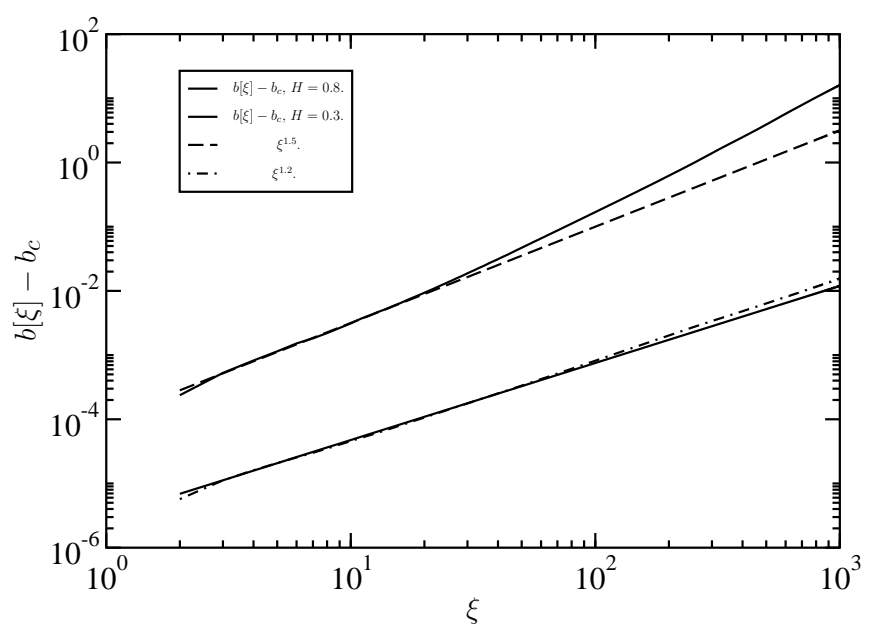

Figure 7: Ordered sequences $b[\xi]-b_{c}$ from 1000 samples of size $1024 \times 1024$ with Hurst exponents $H=0.8$ and $H=0.3$ respectively. The straight lines are proportional to $\xi^{1.2}$ and $\xi^{1.5}$ respectively.

restricitve paths that start at a position $x$,

$$
b(x)=\frac{1}{L}\left[\min _{\mathcal{C}(x)} \int_{\mathcal{C}} d \vec{\ell} \cdot \vec{e}_{\perp} n(\vec{\ell})^{3}\right]^{1 / 3},
$$

where $\mathcal{C}(x)$ is a path starting at $x$. In practice we use a transfer matrix algorithm for this [30]. We have that the most restrictive path is given by $b_{c}=\min _{x} b(x)$.

We may now order the local most restrictive paths, $b(x) \rightarrow b[\xi]$. Fig. 目 shows the ordering statistic of $b[\xi]-b_{c}$ obtained for self affine surfaces $h(x, y)$. For $\xi$ smaller than $50, b[\xi]-b_{c}$ is found to follow a power laws characterised by an exponent $\beta$ close to 1.5 for $H=0.8$ and $\beta=1.2$, for $H=0.3$.

The local most resctrictive path $b(x)$ plays the role of $a+h(x)$ in the one-dimensional case. Under this assumption, the two-dimensional permeability is given by

$$
\frac{L}{K}=\int_{0}^{L} \frac{d x}{k b^{3}(x)}=\int_{0}^{L} \frac{d x}{k\left(\left(b(x)-b_{c}\right)+b_{c}\right)^{3}}
$$

This equation should be compared to Eq. (3) for the onedimensional system. $b_{c}$ in the present case plays the role of $a$ and $b(x)-b_{c}$ plays the role of $h(x)$. Following the logic that led to Eq. (7), we would expect the exponent of the power law of the intermediate regime to be $3-1 / \beta$, hence 2.33 for $H=0.8$ and 2.16 for $H=0.3$. Clearly, the assumption that $b(x)$ could replace $a+h(x)$ in an equivalent system does lead to a reasonable determination of the exponent.

The three regimes are qualitatively illustrated in Fig. 8 that shows the pressure field for different values of $\left(b-b_{c}\right)$. In figure $a$, we see the regime for which the permeability is controlled by a single element - the "AHL" regime, giving rise to a cube law behavior. Figures b and c show the crossover regime giving rise to the intermediate power law in the permeability. The last figure, d, shows the large-opening regime where again a cube law is found. 


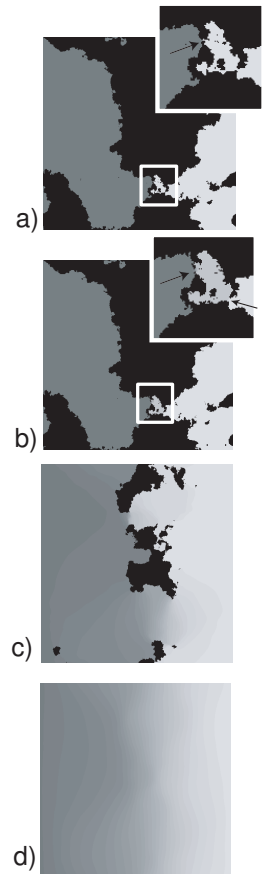

Figure 8: Pressure field in gray levels a self-affine realization. The flow is from left to right. Dark regions correspond to contact zones. From top to bottom: $\left(\left(b-b_{c}\right)=10^{-4}\right.$ ("AHL" regime, the arrow indicates the percolation point), 0.5 (cross over regime, the arrows show the two significant pressure drop corresponding to the two major barriers), 28 (cross over regime) and 97 (cubic law regime).

\section{SUMMARY AND DISCUSSION}

We have in this paper discussed permeability of fractures as a function of fracture opening. We identify three regimes: A first regime where the permeability is completely controlled by one single local area. This area is that identified by the "AHL" construction, and is closely related to the percolation point. In one-dimensional channels, it corresponds to the narrowest constriction. In this regime, we find the permeability follows a cube law with respect to the fracture opening. When the fracture opening is very large, another cube law regime is found.
The prefactors of the two cube laws are different. Between these two regimes, there is an intermediate regime where non-trivial scaling is found. In one-dimensional systems, this scaling can be derived thanks to order statistics for uncorrelated power law aperture distribution. In case of self-affine aperture field, despite long range correlations the order statistics also follows a power law as for the uncorrelated aperture field. Consequently, the three scaling regimes are observed for self affine fractures, with an intermediate exponent of $3-1 / H$.

However, in two dimensions, ordering the permeability distribution modify the effective permeability, ordering statistics is thus no longer applicable in this form. We improved the approach proposed in a previous work 20 where we intoduced the concept of most restrictive path. We used the same concept to modelized the fracture as a sequence of transverse barriers put in series. An hydraulic aperture is then estimated for each barriers. The problem reduce then to a one-dimensional one, where ordering is allowed. We have then shown that the order of each most restrictives barrier displays a power law trend. This model allows us to interpret three observed scaling regimes as function of the equivalent aperture $b_{c}$ of the worst most restrictive path. However, contrary to the one-dimensional case, the scaling law could not be predicted from the roughness of the fracture wall. The obtained exponent in $2 D$ is higher than for the $1 \mathrm{D}$ chanel. This indicates that the deviation to the cubic law is less important in $2 D$ system. This can be understood from the by-pass effect and the localization of the flow. Yet, our approach introduce a new scale $b_{c}$ which allows to continuously describe the crossover from the "AHL" regime to the "cubic law" one. To our knowledge none of the previous methods describe such a behavior.

In future work, other permeability distributions will be considered to generalize our model. A three dimensional extension will also be investigated.

We thank J. P. Hulin for many interesting discussions. A. H. thanks the Université de Paris-Sud 11 for financial support. H. A. and L. T. thanks the PICS "The Physics of Geological Complex System" and the Réseaux de Thématiques de Recherches Avancées "Triangle de la physique" for financial supports.
[1] Committee on Fracture Characterization and Fluid Flow, National Research Council Rock Fractures and Fluid Flow: Contemporary Understanding and Applications, (National Academies Press, Washington, 1996).

[2] K. G. Raven and J. E. Gale, Int. J. Rock Mech. and Mining Science and Geomech. Abs., 22,251 (1985).

[3] P. A. Witherspoon, J. Y. Wang, K. Iway, and J. E. Gale, Water Resours. Res. 16, 1016 (1980).

[4] Y. Méheust and J. Schmittbuhl, Geophys. Lett. 27, 2989 (2000).

[5] V. V. Mourzenko, O. Galamay, J. F. Thovert and P. M. Adler, Phys. Rev. E, 56, 3167 (1997).
[6] P. A. Witherspoon, Geophys. Res. Lett. 8, 659 (1981).

[7] V. V. Mourzenko, J.-F. Thovert and P. M. Adler, Transp. Porous. Media, 45, 89-103 (2001).

[8] S. R. Brown, Geophys. Res. Lett. 13, 1430 (1986).

[9] S. R. Brown, J. Geophys. Res. 92, 337 (1987); ibid. 94, 429 (1989).

[10] S. R. Brown, J. Geophys. Res. B94, 9429 (1989).

[11] R. W. Zimmermann and G. S. Bodvarson, Transp. Porous Media, 23, 1 (1996).

[12] J. B. Walsh, S. R. Brown and W. D. Durham, J. Geophys. Res. B102, 22687 (1997).

[13] G. Drazer and J. Koplik, Phys. Rev. E 66, 026303 (2002). 
[14] J. Inoue and H. Sugita, Water Resours. Res. 39, 1202 (2003).

[15] H. Auradou, G. Drazer, J. P. Hulin and J. Koplik, Water Resours. Res. 41, W09423 (2005).

[16] W. Mallikamas and H. Rajaram, Geophys. Res. Lett., 32, L11401 (2005).

[17] V. Ambegaokar, B. I. Halperin and J. S. Langer, Phys. Rev. B, 4, 2612 (1971).

[18] A. J. Katz and A. H. Thompson, Phys. Rev. B, 34, 8179 (1986).

[19] L. J. Pyrak-Nolte, N. G. W. Cook and D. D. Nolte, Geophys. Res. Lett. 15, 1247 (1988).

[20] L. Talon, H. Auradou and A. Hansen, Water Resour. Res., doi:10.1029/2009WR008404, in press (2010).

[21] V. V. Mourzenko, J-.F. Thovert and P. M. Adler, J. Phys. II France 5465 (1995).

[22] B. B. Mandelbrot, D. E. Passoja and A. J. Paullay, Nature, 308, 721 (1984).
[23] E. Bouchaud, G. Lapasset and J. Planès, Europhys. Lett. 13, 73 (1990).

[24] K. J. Måløy, A. Hansen, E. L. Hinrichsen and S. Roux, Phys. Rev. Lett. 68, 213 (1992).

[25] H. A. David and H. N. Nagaraja, Order Statistics, 3rd Edition (J. Wiley, New York, 2003).

[26] S. Roux, J. Schmittbuhl, J. P. Vilotte and A. Hansen, Europhys. Lett. 23, 277 (1993).

[27] R. Gutfraind and A. Hansen, Transp. Porous Media, 18, 131 (1995).

[28] A. Hansen and S. Roux, J. Phys. A 20, L873 (1987).

[29] A. Hansen and E. L Hinrichsen, Phys. Script. T 44, 55 (1992).

[30] A. L. Barabasi and H. E. Stanley, Fractal Concepts in Surface Growth (Cambridge University Press, Cambridge, 1995). 